\title{
Acknowledgment to reviewers
}

We would like to take this opportunity to acknowledge the time and effort devoted by reviewers to improving the quality of published work in UMUAI. Authors often write to express their appreciation for the detailed and useful comments that we obtain for them. Only rarely we receive complaints, even when the reviews are negative. This is a tribute to the spirit in which reviewing is undertaken for this journal. It is a pleasure, therefore, to be able to pass on thanks from the Editorial Board to the following researchers who also reviewed papers for this year:

Jaewook Ahn, Nick Belkin, Ludovico Boratto, Robbert Beun, Joan-Isaac Biel, Nicola Capuano, Luis M. de Campos, Iván Cantador, Camil Demetrescu, Alexandre Denis, Rebecca Dore, Michael Ekstrand, Zeno Gantner, Mouzhi Ge, Daqing He, Christoffer Holmgård, Michael Kane, Michal Kosinski, Andrej Košir, Kenneth Lachlan, Daniël Lakens, Iolanda Leite, Xin Li, Thomek Loboda, Angeliki Metallinu, Gelareh Mohammadi, Thanasis Mouratidis, Umberto Panniello, Evan Peck, Rainer Planinc, Karen Renaud, Alexander Savi, Olga Santos, David Thomson, Alessandro Vinciarelli, Ivan Vulic, Jason Williams, Yong Zheng. 